\title{
Pérdida dental de los adultos mayores de una corporación Medellín, 2018
}

Dental loss in the elderly population in a private corporation Medellin, 2018

Perda dentária de idoso de empresa de Medellín, 2018

\author{
Catalina González Penagos ${ }^{1}$ \\ Tatiana María Restrepo Ocampo² \\ Diana Verónica Giraldo López ${ }^{3}$ \\ Diana Isabel Ramírez Pérez ${ }^{4}$ \\ Laura Camila Isaza Morales ${ }^{5}$
}

Recibido: 18 de enero de 2020

Aprobado: 5 de agosto de 2020 Publicado: 30 de septiembre de 2020

Cómo citar este artículo: González-Penagos C, Restrepo-Ocampo T, Giraldo-López D, Ramírez-Pérez D, Isaza-Morales L. Pérdida dental de los adultos mayores de una corporación Medellín, 2018. Revista Nacional de Odontología. (2020); 16(1), 1-16. doi: https://doi.org/10.16925/2357-4607.2020.01.09

Artículo de investigación. https://doi.org/10.16925/2357-4607.2020.01.09

1 Institución Universitaria Visión de las Américas. Medellín. Colombia. ORCID: https://orcid.org/0000-0002-7133-891X

Correo electrónico: catalina.gonzalez@uam.edu.co

2 Institución Universitaria Visión de las Américas. Medellín. Colombia.

ORCID: https://orcid.org/0000-0001-5995-0842

3 Institución Universitaria Visión de las Américas. Medellín. Colombia. ORCID: https://orcid.org/0000-0002-2976-9142

$4 \quad$ Institución Universitaria Visión de las Américas. Medellín. Colombia. ORCID: https://orcid.org/0000-0003-1525-9878

5 Institución Universitaria Visión de las Américas. Medellín. Colombia. ORCID: https://orcid.org/0000-0001-5718-7505 


\title{
Resumen
}

Introducción: la falta de funcionalidad física y psíquica del adulto mayor puede desencadenar serios problemas periodontales, caries y lesiones de la mucosa oral, todas estas son patologías que finalmente favorecen la pérdida del nivel de inserción clínica, movilidad dentaria y por último la pérdida dental.

Materiales y métodos: se presenta un estudio analítico transversal, de diseño no experimental con una muestra de 119 adultos mayores institucionalizados, seleccionados por muestreo probabilístico sistemático aleatorio. Resultados: predomina el rango de edad de 67-76 años, el 67,2\% (80) eran solteros y el 40,3\% (48) tenían primaria incompleta. El principal motivo de ingreso a la corporación fue por extrema vulnerabilidad 75,6 \% (90). El 51,3\% (61) de los adultos mayores eran edéntulos totales y el 48,7\% (58) edéntulos parciales, con mayor predomino en los hombres. Perder entre 13 y 24 piezas dentales es un factor de riesgo para tener una clase I de Kennedy en el arco superior (14,700 IC 3551-60.856) e inferior (8,850 IC1378-5682).

Conclusiones: en este estudio se encontraron datos fácilmente comparables con otros autores con respecto a la pérdida dental, sin embargo, es difícil encontrar estudios enfocados en variables sociodemográficas asociadas al edéntulismo, conocimientos y prácticas de autocuidado para los dientes remanentes en el adulto mayor. Es necesario fortalecer el componente de actividades preventivas no solo para los adultos mayores sino a lo largo del ciclo vital.

Palabras clave: adulto mayor, maxilares edéntulos, salud del anciano institucionalizado, salud bucal.

\begin{abstract}
Introduction: The lack of physical and mental functionality in the elderly population, triggers serious periodontal problems, cavities, and lesions in the oral mucosa. These are all pathologies that promote loss of clinical attachment level, tooth mobility, and finally tooth loss.

Materials and methods: Cross-sectional analytical study of non-experimental design with a sample of 119 older adults living in a nursing home, selected through random systematic probabilistic sampling.

Results: Predominantly ages $67-76,67.2 \%$ (80) were single and $40.3 \%$ (48) had an incomplete elementary school education. The main reason for joining a nursing home was because of its extreme vulnerability $75.6 \%$ (90). The $51.3 \%$ (61) of the older adults were totally edentulous and $48.7 \%$ (58) were partially edentulous with a higher prevalence in men. Losing between 13 and 24 teeth is a risk factor for having Kennedy class I in the upper arch (14,700 IC 3551-60,856) and lower arch (8,850 IC1378-5682).

Conclusions: The data, in this study, were easily comparable with other authors regarding tooth loss. However, it is difficult to find studies focused on sociodemographic variables associated with edentulism, knowledge, and self-care practices for remaining teeth in the elderly population. It is necessary to improve the preventive activities not only for older adults but also throughout the life cycle.
\end{abstract}

Key words: elderly, edentulous jaws, health of the institutionalized elderly, oral health.

\section{Resumo}

Introdução: a falta de funcionalidade física e mental do idoso pode desencadear graves problemas periodontais, cáries e lesões da mucosa oral, todas essas patologias que acabam por favorecer a perda do nível de inserção clínica, mobilidade dentária e por fim a perda dentária.

Materiais e métodos: é apresentado um estudo analítico transversal de desenho não experimental com uma amostra de 119 idosos institucionalizados, selecionados por amostragem probabilística sistemática aleatória.

Resultados: predominou a faixa etária de 67 a 76 anos, 67,2\% (80) eram solteiros e 40,3\% (48) tinham o ensino fundamental incompleto. 0 principal motivo de adesão à corporação foi devido à extrema vulnerabilidade 
Catalina González Penagos, Tatiana María Restrepo Ocampo, Diana Verónica Giraldo López, 3 Diana Isabel Ramírez Pérez, Laura Camila Isaza Morales

$75,6 \%$ (90). 51,3\% (61) dos idosos eram edêntulos totais e 48,7\% (58) eram edêntulos parcialmente, com maior prevalência em homens. Perder entre 13 e 24 dentes é um fator de risco para ter classe I de Kennedy na arcada superior (14.700 IC 3551-60.856) e inferior (8.850 IC1378-5682).

Conclusões: neste estudo os dados foram facilmente comparáveis aos de outros autores no que se refere à perda dentária, porém, é difícil encontrar estudos voltados para variáveis sociodemográficas associadas ao edentulismo, conhecimento e práticas de autocuidado para dentes remanescentes em idosos. É necessário fortalecer o componente de atividades preventivas não só para os idosos, mas em todo o ciclo vital.

Palavras-chave: idoso, maxilar desdentado, saúde do idoso institucionalizado, saúde bucal.

\section{Introducción}

En Colombia se considera adulto mayor a las personas con edad igual o superior a los 60 años o mayores de 50 años cuando el deterioro de su estado físico y mental lo determina (1). El DANE, reportó que en el 2013 la población de adultos mayores en Colombia ascendía a los 4.964.793 y en el año 2025 se calcula serán aproximadamente 6.440.000, el 12,6 \% de la población total (2).

En Antioquia, Colombia, la tasa de pobreza de los adultos mayores es de $52 \%$, gran parte de ellos tienen escasos o nulos ingresos, dificultades para acceder a servicios de seguridad social en salud y pensión. Sumado a esto, los casos de agresión también son una situación común, el abandono por parte de sus familiares, el aislamiento y la marginalidad social, son fenómenos frecuentes que se presentan cuando se indaga sobre la calidad de vida de esta población (1, 3).

A todas estas condiciones de vulnerabilidad social, se adiciona el deterioro de la salud presente en esta etapa de la vida, con múltiples modificaciones que alteran de manera progresiva diferentes sistemas, las cuales limitan tanto las facultades físicas como psíquicas del individuo $(4,5)$. Algunos de estos cambios se generan por predisposiciones genéticas, mientras que otros son producto de factores ambientales (2).

Estas limitaciones condicionan la capacidad del adulto mayor para llevar a cabo su cuidado personal básico o cumplir tareas que se deben realizar todos los días (3). Tareas cotidianas que necesitan de la ayuda de otro para llevarse a cabo y que progresivamente crean la necesidad de dependencia del adulto mayor para poder subsistir $(3,6)$.

Dentro de estas actividades habituales y necesarias para la vida, el cuidado de la salud bucal es fundamental para garantizar una adecuada alimentación y prevenir síntomas dolorosos asociados a los tejidos bucales. Sin embargo, estos cuidados fundamentales se ven limitados por la disminución de la destreza psicomotora, una condición frecuente en el adulto mayor, lo que desencadena serios problemas periodontales, caries y lesiones de la mucosa oral, todas estas son patologías que 
finalmente favorecen la pérdida del nivel de inserción clínico, movilidad dentaria y por último la pérdida dental (7).

Por otro lado, existe otra situación que pone en riesgo la salud oral de los adultos mayores y es la ingesta de medicamentos. A pesar de que los fármacos son una herramienta terapéutica para mejorar la calidad de vida, su uso no está exento de efectos adversos. En el presente son muchos los adultos mayores polimedicados y existe un amplio número de fármacos que pueden dar lugar a numerosos efectos adversos en la cavidad bucal. Algunos son: xerostomía, alteraciones del gusto, agrandamiento gingival, mucositis, alteraciones de las glándulas salivales, pigmentaciones, halitosis, entre otros (8).

Adicional a la falta de motricidad para una adecuada higiene oral y el uso de medicamentos, los tejidos de la cavidad bucal comienzan a presentar cambios fisiológicos. Esta característica los hace más susceptibles al acumulo de placa y aumentan la prevalencia de las patologías anteriormente mencionadas, dejando como consecuencia una mayor pérdida de estructuras dentales. (9)

Esta condición de edentulismo tan representativa para los adultos mayores, en muchas ocasiones no es resuelta, los dientes perdidos no son reemplazados por la falta de recursos económicos y en algunos casos cuando son reemplazados, el adulto no se adapta al uso de la prótesis dental. La falta de solución a esta condición genera problemas graves en funciones vitales como la masticación, nutrición, además de problemas en la calidad de vida $(10,11)$.

La relación entre una adecuada eficiencia masticatoria y el número de dientes presentes en boca, es una asociación que está bien documentada en la literatura. La pérdida de dientes posteriores con sus antagonistas genera alteraciones en la trituración de los alimentos, fenómeno que progresivamente determina el estado de nutrición del individuo. Los adultos mayores que presentan pérdida dental refieren una dieta limitada en proteínas, frutas y vegetales, restringiendo su dieta a alimentos más fáciles de masticar, pero con alto valor energético y bajo valor nutricional $(9,10)$.

Estos inconvenientes relacionados a la pérdida dental se pueden prevenir desde el autocuidado con buenas técnicas de higiene oral. Sin embargo, gran cantidad de adultos mayores siguen aceptando que el deterioro bucal del aparato masticatorio es un proceso inevitable e irreversible del envejecimiento, lo que causa el descuido de su salud bucal (12). Por otro lado, en muchas circunstancias, por la falta de funcionalidad física y psíquica del adulto mayor, esta tarea queda a cargo o es supervisada por un cuidador, la mayor parte de esta población es asistida por algún miembro del vínculo familiar (13-15) y en otros casos, cuando el adulto mayor es internado en hogares geriátricos, por un cuidador con un nivel de formación para su asistencia. Sin 
Catalina González Penagos, Tatiana María Restrepo Ocampo, Diana Verónica Giraldo López, 5 Diana Isabel Ramírez Pérez, Laura Camila Isaza Morales

embargo, investigaciones evidencian que generalmente estas personas tienen una capacitación muy limitada sobre el cuidado de la salud oral en pacientes geriátricos $(13,16,17)$.

Esta situación genera preocupación con respecto al bienestar del adulto mayor institucionalizado, principalmente en el contexto de este estudio, en el cual la corporación brinda una vivienda permanente o temporal y es responsable de ofrecer un cuidado integral. Sin embargo, no cuenta con los recursos para un seguimiento del estado bucodental, un diagnóstico o un tratamiento odontológico (18).

El objetivo general de este estudio fue determinar la pérdida dental de los adultos mayores institucionalizados de una corporación Medellín, 2018.

\section{Materiales y métodos}

Estudio analítico transversal, de diseño no experimental realizado de mayo a octubre de 2018, en una corporación de adultos mayores de la ciudad de Medellín.

La población estuvo conformada por 172 adultos mayores. Para la obtención de la muestra se realizó un muestreo probabilístico con un nivel de confianza del 95 \%, un margen error del 5 \% y una proporción esperada del 50 \%, para la selección de los participantes se aplicó un muestreo sistemático aleatorio. De esta manera la muestra quedó conformada por 119 adultos. Se contó con la solicitud previa del consentimiento informado, garantizando el respeto y la confidencialidad en la información.

Se elaboró un instrumento tipo encuesta estructurada, que incluía variables sociodemográficas y variables que permitieron la identificación de la pérdida dental. Finalmente, estos pacientes fueron examinados por una odontóloga con la asistencia de dos auxiliares, en las instalaciones de la corporación, en un espacio abierto, iluminado y ventilado ayudado por instrumental básico para encontrar los hallazgos estomatológicos.

Posterior a la recolección de la información se elaboró una base de datos en el programa Excel y fue exportado al paquete estadístico PRISM® (versión 6.07 junio 12 de 2015) con licencia de la Fundación Universitaria Autónoma de las Américas. Se calcularon frecuencias simples, tablas de 2 por 2 y el valor de p de significancia menor a 0.05 (19).

Este estudio estuvo acorde con la normativa nacional para investigación en seres humanos, según la Resolución 8430 de 1993 del Ministerio de Protección Social y Salud (20). Se considera una investigación de riesgo mínimo, el proyecto contó con el aval del Comité de Ética en Investigación institucional (CEI) (Acta 36 del 13 de septiembre de 2017). 


\section{Resultados}

Para esta investigación, se encontró un predominio de hombres en un 60,5 \% (72), el 39,5 \% (47) restante eran mujeres. La edad media de los adultos mayores fue de 70,8 y una desviación estándar de 9,73 años. El 67,2 \% (80) eran solteros, seguido del 13,4 \% (16) que eran viudos, el 19,3\% (23) tenían un estado civil entre casado, divorciado y separado. Los porcentajes más altos encontrados frente al nivel de escolaridad fue el 40,3 \% (48) tenían primaria incompleta y el 38,6 \% (46) primaria completa y sin educación. El principal motivo de ingreso a la corporación fue por extrema vulnerabilidad 75,6 \% (90) seguido de la condición en situación de calle 19,3 \% (23) y abandono de la familia 4,2\% (5).

Con respecto a la pérdida dental, el 51,3 \% (61) de los adultos mayores eran edéntulos totales y el 48,7 \% (58) edéntulos parciales. El 58 \% (69) tenían una pérdida de 29 a 32 dientes permanentes, seguido del 19,3\% (23) con una pérdida de 21 a 25 dientes, el 5,9\% (7) con una pérdida de 26 a 28 dientes y el 16,8 \% (20) tenían una pérdida de 20 dientes o menos.

Se encontró una pérdida dental del 83,4 \% (3177), solo el 16,6 \% (631) de los dientes permanentes se conservaban en boca. La pérdida por grupo de dientes fue mayor en los molares en un 40,5 \% (1287), seguido de los premolares 24,5 \% (779), incisivos $24,4 \%$ (775) y finalmente el grupo de los caninos 10,6 \% (336).

Por maxilares se pudo evidenciar, mayor pérdida dental en el arco superior, de un 52,5\% (1669) con respecto al arco inferior 47,5\% (1508). En este estudio, el $37 \%$ (44) de los adultos mayores tenían prótesis, mientras que el 63 \% (75) refirió no portar prótesis. En el maxilar superior el 28,6 \% (34) usan prótesis total removible, en tanto el 5,9 \% (7) de los adultos usa prótesis parcial removible y el 0,8 \% (1) usa prótesis fija. El $64,7 \%(77)$ de los adultos mayores no utiliza prótesis superior.

Con respecto al maxilar inferior el 19,3 \% (23) usa prótesis total removible, en tanto el 4,2 \% (5) usan prótesis parcial removible y el 76,5 \% (91) no utiliza prótesis inferior. Dado que hay personas que usan prótesis bimaxilar, el número total de prótesis es de 70 .

Con respecto a los adultos mayores edéntulos totales 51,3 \% (61) y parciales 48,7 \% (58) y teniendo en cuenta para la clasificación de Kennedy solo a la población de adultos mayores edéntulos parciales, se halló una mayor prevalencia de espacios edéntulos en el maxilar inferior, esto a pesar de que la perdida de dientes fue mayor en el maxilar superior, lo que coincide con un mayor número de arcos superiores totalmente edéntulos $64,7 \%$ (77) en contraste con un 53,8 \% (64) de arcos inferiores totalmente edéntulos. 
Catalina González Penagos, Tatiana María Restrepo Ocampo, Diana Verónica Giraldo López, 7 Diana Isabel Ramírez Pérez, Laura Camila Isaza Morales

En la clasificación de Kennedy los maxilares parcialmente edéntulos obtuvieron los siguientes resultados: en el arco superior el 17,7 \% (21) son clase I, el 8,4 \% (10) son clase II, el 8,4 \% son clase III (10) y el 0,8 \% (1) es clase IV; el 64,7\% (77) de los maxilares superiores restantes no se tuvieron en cuenta por ser totalmente edéntulos. Con respecto a los maxilares inferiores, el 32,8 \% (39) son clase I, el 7,5 \% (9) son clase II y el 5,9\% (7) son clase III, el 53,8\% (64) restante no se tuvo en cuenta por tratarse de maxilares totalmente edéntulos (tabla 1).

Tabla 1. Resultados de la clasificación de Kennedy en la población de los adultos mayores de una corporación. Medellín, 2018

\begin{tabular}{lcc}
\hline \multicolumn{1}{c}{ Variable } & Frecuencia & (\%) \\
\hline Maxilar superior & 21 & 17,7 \\
\hline Clase I & 10 & 8,4 \\
\hline Clase II & 10 & 8,4 \\
\hline Clase III & 1 & 0,8 \\
\hline Clase IV & & 32,8 \\
\hline Maxilar inferior & 39 & 7,5 \\
\hline Clase I & 9 & 5,9 \\
\hline Clase II & 7 & 0 \\
\hline Clase III & 0 & \\
\hline Clase IV & 9 & \\
\hline
\end{tabular}

Fuente: elaboración propia

Las piezas dentales presentes en boca solo fueron el 16,6 \% (631), de las cuales se encontró que el 59,43 \% (375) de los dientes estaban sanos, el 11,25 \% (71) tenían restauraciones en buen estado, el 0,32 \% (2) eran pilares para coronas completas fijas individuales en buen estado, sin embargo, el 15,85 \% (100) estaban indicados para extracción, el 12,99 \% (82) presentaron restauraciones defectuosas y el 0,16 \% (1) se trataba de un pilar con corona individual fija en mal estado; lo que indica una necesidad de tratamiento para el 28,7 \% (181) de los de los 631 dientes presentes en boca y un porcentaje de pérdida dental susceptible de continuar aumentando.

\section{Análisis bivariado}

Al llevar a cabo el análisis bivariado, se encontró asociación estadísticamente significativa entre la edad y el edentulismo $p=0,012$. El 26,1 \% (31) de los adultos que tienen entre 67 y 76 años, tuvieron una pérdida dental de 29 a 32 dientes, encontrándose una significancia de $p=0,015$. 
No se encontró asociación entre la pérdida dental con el sexo $(p=0,402)$, nivel de escolaridad $(p=0,367)$ y estado civil $(p=0,919)$, son los adultos mayores solteros quienes tienen mayor pérdida 67,2\% (80) que aquellos que se encuentran viudos. Se pudo observar que quienes reportaron tener primaria incompleta y aquellos sin nivel educativo fueron quienes presentaron mayor pérdida dental, sin embargo, no se encontró asociación significativa.

Se halló que la pérdida dental asociada al sexo (UMA 1407,500 sig. 0,088) no es significativo. Sin embargo, la muestra no se encuentra en una relación 1:1, porque son más adultos mayores hombres que mujeres, lo que no permitió realizar asociaciones entre perdida dental y sexo. Con respecto a la clasificación de Kennedy superior e inferior, con la pérdida dental, se halló asociación estadísticamente significativa p=0,000.

En este grupo poblacional existe una asociación 0,000 y correlación 0,000 cuando se pierden pocas piezas dentales (entre 1 y 4). El diente que generalmente se pierde en el cuadrante 1, es el segundo premolar superior derecho (diente 15), en el cuadrante dos es el tercer molar superior izquierdo (diente 28), en el cuadrante tres son el primer premolar inferior izquierdo (diente 35), primer, segundo y tercer molar inferior izquierdo (dientes $36,37,38$ ) y en el cuadrante cuatro son los dientes incisivo lateral inferior derecho (diente 42) y segundo y tercer molar inferior derecho (diente 47 y 48$)$.

\section{Multivariado}

Perder entre 13 y 24 piezas dentales aumenta la probabilidad de tener una clase I de Kennedy en el arco superior (14,700 IC 3551-60.856) e inferior (8,850 IC1378-5682), Io que indica que los primeros dientes que se pierden son los posteriores.

\section{Discusión}

En este estudio se encontró un mayor predominio de hombres 60,5\% esta distribución de sexo coincide con la reportada en algunos estudios de adultos mayores institucionalizados en Villavicencio 69,1 \% hombres (18) y en Bucaramanga 55 \% hombres (21). Sin embargo, estas proporciones en sexo difieren de los reportes nacionales del DANE, en los cuales la proporción de mujeres adultas mayores es superior comparada con la de los hombres. Aumentos sostenidos desde los años setenta hasta las proyecciones para el año 2020 (22). Sin embargo, es importante tener en cuenta que los datos del DANE, no hacen referencia solo a la población institucionalizada, e 
Catalina González Penagos, Tatiana María Restrepo Ocampo, Diana Verónica Giraldo López, 9 Diana Isabel Ramírez Pérez, Laura Camila Isaza Morales

incluyen a mujeres adultas mayores que no se encuentran en situación de vulnerabilidad y por tanto no hacen parte de la población institucionalizada.

Se encontró, además, un mayor porcentaje de población soltera 67,2%, hallazgo que corresponde con los antecedentes de extrema vulnerabilidad y abandono que presentan los participantes de este estudio. Situación que difiere a los reportes con población no institucionalizada, en la que predominan individuos casados, Bellamy y Moreno (23) 69,6 \% Cárdenas y Arrieta 49,1 \% (24) y el estudio de Cárdenas y Velázquez (25) 58,9\%. En estos últimos estudios es importante destacar que el principal cuidador es el conyugue.

Este último aspecto ha tenido relevancia en otros trabajos como el publicado por Islas y Medina (26) en población mexicana institucionalizada y no institucionalizada, en el que se reporta una menor pérdida dental para individuos que convivían con su pareja 25 \% (21 dientes o más), comparados con aquellos que convivían en asilos 11,5\% (menos de 21 dientes en boca). Cárdenas y Velázquez (25) relacionan la convivencia como factor que fomenta las prácticas de autocuidado refiriendo que los participantes que no vivían solos fueron quienes obtuvieron una mejor autopercepción de salud bucal.

Al comparar los resultados del actual estudio, con población de estrato socioeconómico bajo, pero con mayor estabilidad económica y emocional, como es el caso de individuos pensionados, con casa propia, que conviven con un tipo de familia nuclear, los porcentajes de pérdida dental en estas condiciones se ven reducidos. En el estudio de Cárdenas y Arrieta (24) se cumplieron estas condiciones, reportándose en un $75 \%$ de la población menos de 19 dientes en boca. Comparados con el $83 \%$ de individuos que en este estudio presentaron menos de 12 dientes en boca. Cárdenas y Arrieta explican ante este fenómeno que las condiciones económicas son fundamentales en la vejez para poder acceder a cuidados dignos que conlleven a mejores condiciones de salud bucal $(24,27)$.

Con respecto a los hallazgos encontrados según el nivel de escolaridad, es evidente la escaza formación académica de este grupo poblacional, el 40,3\% reporta primaria incompleta y el 19,3 \% manifiesta no tener ningún tipo de educación. Estos datos son similares a los encontrados por Alonso y Ríos (26) en adultos mayores de Barranquilla, donde el mayor porcentaje reportó tener primaria incompleta 52,5\% y el 12,5 \% ningún tipo de formación. En el estudio de Monroy y Méndez (28) en Bogotá el 64,7 \% reportó alcanzar un nivel de primaria y el 11,2 \% no tuvo acceso a educación. En este último estudio solo el 17 \% de los participantes presento 21 o más dientes en boca, en contraste con el presente estudio 12 dientes o menos. 
Autores como Cárdenas y Arrieta (24) explican que la baja escolaridad encontrada en los adultos mayores es un fenómeno asociado al poco acceso presentado hace sesenta años a servicios como la salud, el trabajo y la educación.

En la valoración clínica los resultados encontrados en este estudio, reportan que el $100 \%$ de los adultos mayores tenían antecedentes de pérdida dental. Estos porcentajes son iguales a los obtenidos por Rodas y Angarita (18) en otras zonas de Colombia, en Villavicencio $100 \%$, en estudios de población latina en Chile (29) $100 \%$, y en México (23) $97 \%$. Esta cifra se aproxima a la reportada en el ENSAB IV, donde el grupo de edad entre los 65 a los 79 años tienen un 98,9\% de pérdida dental (30).

El 51,3\% de los adultos mayores evaluados en esta investigación eran totalmente edéntulos y el $48,7 \%$ parcialmente edéntulos. Resultados similares al estudio realizado por Rodas y Angarita (18), en una población de adultos mayores donde reportaron la presencia de edéntulismo total en un 48,5 \% y edéntulismo parcial en un $51,5 \%$. El parecido entre estos estudios posiblemente se deba a que fueron realizados en población colombiana e institucionalizada.

No obstante, los resultados en población latina no institucionalizada difieren de este estudio, reportando un edéntulismo total de 9,9\% en un estudio en México (31) y el 13,7\% en Chile (29). Igualmente, los porcentajes de edéntulismo encontrados en población no institucionalizada a nivel nacional en el ENSAB IV, son menores a los reportados en este estudio, el 7,43 \% de la población presenta edéntulismo total bimaxilar entre los 45 a 64 años y alcanza el 32,8 \% entre los 65 y 79 años (30).

Además, se encontró un promedio de dientes perdidos más alto en hombres, resultados relativamente similares a los reportados en Chile por Arteaga y Espinoza (29) y por Rodas y Angarita en Villavicencio (18), ambos estudios realizados en población mayor de 65 años. Las diferencias estadísticamente significativas entre hombres y mujeres respecto a piezas pérdidas son explicadas por Rodas y Angarita. Estas van desde las políticas de salud que privilegian la atención de la mujer embarazada y la población infantil en el sector público, mientras que los hombres que logran acceder a servicios de salud, en este caso atención dental, asisten generalmente por urgencia para la extracción de sus piezas dentarias (29).

Islas y Medina también encontraron mayor pérdida dental en hombres mayores de 60 años, los autores consideran que los hombres estuvieron principalmente expuestos a servicios de salud bucal de tipo mutilante. En contraste con las mujeres, quienes tuvieron más probabilidad de no extraerse los dientes por motivos estéticos o por conductas de tipo restaurador en donde prefieren un tratamiento más conservador que radical (32). 
Catalina González Penagos, Tatiana María Restrepo Ocampo, Diana Verónica Giraldo López, 11 Diana Isabel Ramírez Pérez, Laura Camila Isaza Morales

Sin embargo, estudios realizados en población más joven, concluyeron que no había diferencias estadísticamente significativas entre pérdida dental y género. Es el caso del estudio realizado por Vanegas y Villavicencio (33), en una población mayor de 18 años, y el estudio realizado en México por Fernández y Medina (31) en una población mayor de 35 años.

El $83 \%$ de la población de este estudio tuvo una pérdida dental entre 21 y 32 dientes, indicando que es una población sin funcionalidad adecuada, teniendo en cuenta la definición de la OMS para la dentición funcional como la presencia de 21 o más dientes en boca excluyendo prótesis (28).

En otros estudios también se han observado porcentajes altos de pérdida dental, con hallazgos similares al presente estudio. Como el de Arteaga y Espinoza (29), donde el promedio de dientes perdidos por individuo fue de 20,52 y el estudio de Joya y Quintero (34), en el cual el promedio de dientes en boca para cada individuo es de 11. Esta cantidad tan escasa de dientes es compatible con los resultados del ENSAB $I V$, donde se encontró que los adultos mayores entre 65 y 79 años tenían un promedio de dientes perdidos de 20 (30).

Cabe destacar que estos tres últimos estudios fueron realizados en población no institucionalizada mayor de 65 años, y en el presente estudio la población es más joven, mayor de 56 años e institucionalizada, sin embargo, los resultados de pérdida dental son mayores, con un promedio de 26 dientes perdidos y 6 dientes en boca. Si se contrastan los hallazgos con estudios realizados en adultos mayores de 50 años no institucionalizados, como es el caso del trabajo de Bellamy y Moreno (31) 16,9 dientes presentes en boca, se evidencia que en este estudio la pérdida dental es mayor.

Con respecto a la clasificación de Kennedy, los datos reportados en este estudio evidencian una mayor frecuencia de casos para la clase I en ambos maxilares. Resultados que difieren a los registrados en un estudio en Ecuador (33), donde la clase III fue predominante en ambos maxilares (42\% maxilar superior y $40 \%$ inferior). De igual manera en un estudio en Villavicencio (14) la clase III fue predominante en el maxilar superior $(7,3 \%)$, mientras que en el maxilar inferior predominó la clase I $(22,1 \%)$, en este último aspecto hay coincidencia con este estudio.

Según el uso de aparatología en boca, el $37 \%$ de las personas presentaban prótesis, cifra muy similar a la reportada en un estudio en México, donde el $35 \%$ de los individuos utilizaban prótesis (23). En un estudio en Chile un 38,5\% de la población utilizaba prótesis (29) y cifras encontradas en Villavicencio $47 \%$ de los adultos mayores portaban prótesis (30). En todos estos estudios se evidencia un mayor porcentaje de uso de prótesis total removible, seguido de prótesis parcial removible y por último 
están los usuarios de prótesis fija. En este orden también hay coincidencia con el presente estudio.

Frente a la necesidad real de prótesis que presenta la población de este estudio, los porcentajes de uso de prótesis son bajos comparados con los hallazgos de pacientes edéntulos parciales y totales. Situación que se hace evidente al comparar los porcentajes con lo reportado en el ENSAB IV, donde la población de 65 a 79 años alcanza el 77,4 \% para el uso de alguna aparatología protésica (30). Algunos autores, como es el caso de Bellamy y Moreno (31) explican este fenómeno asociado a los bajos ingresos económicos y el elevado costo del tratamiento rehabilitador protésico, tomando en consideración que este tipo de procedimientos no está incluido por los servicios de salud pública, además de los límites de cubrimientos de seguridad social en salud para esta población.

\section{Conclusiones}

En este estudio se encontraron datos fácilmente comparables con otros autores con respecto a la pérdida dental, que ha sido un tema bien desarrollado y con una amplia información en los trabajos de investigación consultados, sin embargo, es difícil encontrar estudios enfocados en otros aspectos igualmente relevantes, como las variables sociodemográficas asociadas al edéntulismo y los conocimientos y prácticas de autocuidado para los dientes remanentes en el adulto mayor. Por tanto, es necesario fortalecer el componente de actividades preventivas no solo para los adultos mayores sino a lo largo del ciclo vital, para contrarrestar los efectos de la pérdida dental en las nuevas generaciones. Esto supondría que en el futuro los adultos mayores tendrían unas mejores condiciones de salud bucal.

El presente estudio tiene algunas limitaciones que conviene considerar al analizar los hallazgos. Por ejemplo, la población se limita a una comunidad de adultos mayores institucionalizados en un contexto especifico que, si bien representan características sociodemográficas semejantes a otros adultos mayores, en algunos aspectos analizados no presentan coincidencia. Es el caso de la relación con respecto al sexo, situación que no permitió realizar asociaciones y comparaciones con la pérdida dental, modificándose la proporción, incluso al compararla con las cifras del DANE, las cuales tienen en cuenta a toda la población de adultos mayores, tanto institucionalizados como no institucionalizados a nivel nacional.

Sin embargo y pese a la falta de representatividad estatal, este estudio constituye una base de datos útil que permite abrir más espacios para continuar generando conocimiento y propuestas que conduzcan a la formulación de políticas públicas, 
Catalina González Penagos, Tatiana María Restrepo Ocampo, Diana Verónica Giraldo López, 13 Diana Isabel Ramírez Pérez, Laura Camila Isaza Morales

programas educativos y sociales que respondan a las necesidades de los adultos mayores con el objetivo de mejorar sus condiciones de salud bucal y su calidad de vida en los próximos años.

\section{Referencias}

1. Cardona J, Álvarez M, Pastrana S. Calidad de vida relacionada con la salud en adultos mayores de hogares geriátricos. Rev Cienc Salud. 2014; 12 (2): 139-155. doi: dx.doi.org/10.12804/ revsalud12.2.2014.01

2. Laguado E, Camargo K, Campo Torregroza E, Martín M. Funcionalidad y grado de dependencia en los adultos mayores institucionalizados en centros de bienestar. Gerokomos. 2017; 28 (3): 135-141. Recuperado en: http://scielo.isciii.es/scielo. php?script=sci_arttext\&pid=S1134-928X2017000300135\&lng=es.

3. Huenchuan S, Rodríguez R. Necesidades de cuidado de las personas mayores en la ciudad de México. Santiago de Chile. 2015 Recuperado en: https://repositorio.cepal.org/bitstream/ handle/11362/38879/S1500754_es.pdf?sequence=1\&isAllowed=y

4. Reques P. El análisis demográfico del envejecimiento: conceptos, técnicas y métodos. Santander: Servicio de publicaciones de la Universidad de Cantabria. 2011. Recuperado en: https://ocw.unican.es/mod/page/view.php?id=704

5. Franco González Lina María, Escobar Restrepo Germán David, Turizoa Rovira Arleth Patricia, Vélez Ceballos Margarita, Cardona Gil Diana Lucia. Des-cripción de cambios clínicos de las mucosas orales en adultos mayores. Revista Nacional de Odontología. 2011; 7(12): 28-34.

6. Dorantes G, Ávila J, Mejía S, Gutiérrez L. Factores asociados con la dependencia funcional en los adultos mayores: un análisis secundario del Estudio Nacional sobre Salud y Envejecimiento en México. Rev Panam Salud Pública. 2007; 22(1): 3-11. doi: 10.1590/ S1020-49892007000600001

7. Chávez B, Manrique J. Odontogeriatría y Gerodontología: el envejecimiento y las características bucales del paciente adulto mayor: revisión de literatura. Rev Estomatol Herediana. 2014 [citado 2019 Jul 2]; 24 (3): 199-207. Recuperado en: http://www.upch.edu.pe/vrinve/dugic/ revistas/index.php/REH/article/view/2096/2086. DOI: 10.20453/reh.2014.2096 
8. Bascones A, Muñoz M, Bascones C. Reacciones adversas a medicamentos en la cavidad oral. Medicina Clínica Elsevier. 2015; 144 (3):126-131. doi: 10.1016/j.medcli.2014.01.025

9. Díaz S, Arrieta K, Ramos K. Impacto de la Salud Oral en la Calidad de Vida de Adultos Mayores. Rev Clin Med Fam. 2012; 5 (1): 9-16. doi: 10.4321/S1699-695X2012000100003

10. Mesas E, Andrade M, Marcos S, Bueno L. Salud oral y déficit nutricional en adultos mayores no institucionalizados en Londrina, Paraná, Brasil. Rev. bras. epidemiol. 2010; 13 (3): 434-445. doi: 10.1590/S1415-790X2010000300007

11. Gaitán HF, Sánchez-Mendoza F, González-Colmenares G. Barreras físicas y estructurales para el tratamiento odontológico de personas con discapacidad motora. Rev Nac Odontol. 2013: 9(17): 41-46. doi: https://doi.org/10.16925/od.v9i17.574

12. Cerón-Bastidas XA. Calidad de vida y su relación con la salud oral en personas de la tercera edad. Rev Nac Odontol. 2014; 10(19):83-9. doi: http://dx.doi.org/10.16925/od.v10i19.853

13. Fuentes C, Escobar C, Muñoz P, Ottone N. Creencias en Salud Oral de Cuidadores de Pacientes Discapacitados y Adultos Mayores Institucionalizados de la Comuna de Quilaco, Región del BioBío. Int. J. Odontostomat. 2015; 9 (2): 301-306. doi: 10.4067/S0718-381X2015000200018

14. Hernández Z. Cuidadores del adulto mayor residente en asilos. Index Enferm. 2006; 15 (5253): 40-44. doi: 10.4321/S1132-12962006000100009

15. Zabalegui A, Juando C, Izquierdo M, Gual P. Recursos y consecuencias de cuidar a las personas mayores de 65 años: una revisión sistemática. Gerokomos. 2004; 15 (4): 199-208. Recuperado en: https://www.gerokomos.com/wp-content/uploads/2015/01/15-4-2004-199-rincon.pdf

16. Garrido C, Espinoza I, Romo F. Traducción al español y validación del cuestionario de creencias en salud bucal para cuidadores (DCBS-SP). Rev. Esp. Salud Pública. 2010; 84 (4): 409-416. doi: 10.1590/S1135-57272010000400005

17. Luengo C, Araneda G, López M. Factores del cuidador familiar que influyen en el cumplimiento de los cuidados básicos del usuario postrado. Index Enferm. 2010; 19 (1): 14-18. doi: 10.4321/S1132-12962010000100003

18. Rodas C, Angarita M, Nemocon L, Pinzón L, Robayo Y, González R. Estado bucodental de adultos mayores institucionalizados mediante un programa público en Villavicencio, durante el primer semestre de 2014. Revista Investigaciones Andina. 2016; 18 (33): 1625-1646. doi: $10.33132 / 01248146.646$ 


\section{Catalina González Penagos, Tatiana María Restrepo Ocampo, Diana Verónica Giraldo López, 15 Diana Isabel Ramírez Pérez, Laura Camila Isaza Morales}

19. Londoño F. Metodología de la investigación epidemiológica. $5^{a}$ ed. Bogotá: Manual Moderno; 2014.

20. Resolución 8430 de 1993 (En línea). Ministerio de salud. (Consulta: Dic 5 de 2017). Recuperado en: https://www.minsalud.gov.co/sites/rid/Lists/BibliotecaDigital/RIDE/DE/ DIJ/RESOLUCION-8430-DE-1993.PDF.

21. BermúdezWilliamFernando,ConchaSánchezSoniaConstanza,Camargo LemosDianaMarina. Perfil orofacial de las personas mayores institucionalizadas de la ciudad de Bucaramanga y su área metropolitana. USTASALUD. doi: https://doi.org/10.15332/us.v2i1.1849

22. República de Colombia. Ministerio de salud y Protección Social. Envejecimiento demográfico. Colombia 1951-2020 dinámica demográfica y estructuras poblacionales. 2013; 48p. Recuperado en: https://www.minsalud.gov.co/sites/rid/Lists/BibliotecaDigital/RIDE/DE/PS/ Envejecimiento-demografico-Colombia-1951-2020.pdf

23. Bellamy Ortiz C.l., Moreno Altamirano A. Relación entre calidad de vida relacionada con la salud oral, pérdida dental y prótesis removible en adultos mayores de 50 años derechohabientes del IMSS. Avances en Odontoestomatología. doi: 10.4321/S0213-12852014000400003

24. Díaz Cárdenas Shyrley, Arrieta Vergara Katherine, Ramos Martínez Ketty. Impacto de la Salud Oral en la Calidad de Vida de Adultos Mayores. Rev Clin Med Fam. 2012 Feb; 5(1): 9-16. doi: 10.4321/S1699-695X2012000100003

25. Cárdenas Bahena Ángel, Velázquez Olmedo Laura Bárbara, Falcón Flores Laura Bárbara, García Zámano Itzel Estela, Montes Salmerón Rosa Elena, Reza-Bravo Gustavo Gil, Sánchez García Sergio. Autopercepción de la salud oral en adultos mayores de la Ciudad de México. Rev Med Inst Mex Seguro Soc. 2018; 56 (1): 54-63. Recuperado en: https://www.medigraphic. com/pdfs/imss/im-2018/ims181h.pdf

26. Alonso Palacio, LM, Ríos, AL, Payares, SCd, Maldonado, A, Campo, L, Quiñonez, D, Zapata, Y. Percepción del envejecimiento y bienestar que tienen los adultos mayores del Hogar Geriátrico San Camilo de la ciudad de Barranquilla (Colombia). Salud Uninorte. 2010; 26(2):250-259. Recuperado en: http://www.redalyc.org/articulo.oa?id=81719006007

27. Wong R., Espinoza M. Adulto Mayor Mexicano en el Contexto Socioeconómico Amplio: Salud y Envejecimiento. Salud Pública de México. 2007; 49 (4): 436-446. Recuperado en: http://envejecimiento.sociales.unam.mx/articulos/1-salud.pdf 
28. Monroy Ramírez MJ, Méndez Castilla JM, Tello Medina MA, Buitrago-Medina DA. Factores asociados a dentición funcional en adultos mayores de la subred centro oriente, Bogotá, 2017. Rev. CES Odont. 2018;; 31(2): 15-27. doi: http://dx.doi.org/10.21615/ cesodon.31.2.2

29. Arteaga O., Urzúa I., Espinoza I., Muñoz A. y Mendoza C. Prevalencia de Caries y Pérdida de Dientes en Población de 65 a 74 Años de Santiago, Chile. Rev. Clin. Periodoncia Implantol. Rehábil. Oral. 2009;; 2 (3): 161-166. Recuperado en: https://www.sciencedirect.com/science/ article/pii/S0718539109700278

30. República de Colombia. Ministerio de salud. IV Estudio Nacional de Salud Bucal ENSAB IV 2013-2014. 2015;378p.. Recuperado en: https://www.minsalud.gov.co/sites/rid/Lists/ BibliotecaDigital/RIDE/VS/PP/ENSAB-IV-Situacion-Bucal-Actual.pdf

31. Fernández Barrera Miguel Á., Medina Solís Carlo E., Márquez Corona María de L., Vera Guzmán Sergio, Ascencio Villagrán Arturo, Minaya Sánchez Mirna y Casanova Rosado Alejandro J. Edentulismo en adultos de Pachuca, México: aspectos sociodemográficos y socioeconómicos. Revista Clínica de Periodoncia, Implantología y Rehabilitación Oral. 2016;; 9 (1): 59-65. Recuperado en: https://www.sciencedirect.com/science/article/pii/S0718539116000057 https://doi.org/10.1016/j.piro.2015.12.004

32. Islas Granillo Horacio, Medina Solís Carlo E., Navarrete Hernández José J., Minaya Sánchez M., Vallejos Sánchez Ana A., Fernández Barrera Miguel Á. y Cuevas Suarez Carlos E. Prevalencia de dentición funcional en ancianos mexicanos. Rev Clin Periodoncia Implantol Rehabil Oral. 2015;; 8(2): 150-156. doi: 10.1016/j.piro.2015.04.004

33. Vanegas Avecillas Elizabeth, Villavicencio Caparó Ebingen, Alvarado Jiménez Omar, Ordóñez Plaza Patricia. Frecuencia del edentulismo parcial y total en adultos y su relación con factores asociados en la clínica universitaria Cuenca Ecuador 2016. Rev Estomatol Herediana. 2016 Octubre-Dic; 26 (4):215-220. doi: https://doi.org/10.20453/reh.v26i4.3027

34. Joya LD, Quintero LE. Caracterización de la salud bucal de las personas mayores de 65 años de la ciudad de Manizales, 2008. Hacia promoc. salud. 2015; 20(1): 140-152. doi: 10.17151/ hpsal.2015.20.1.10 